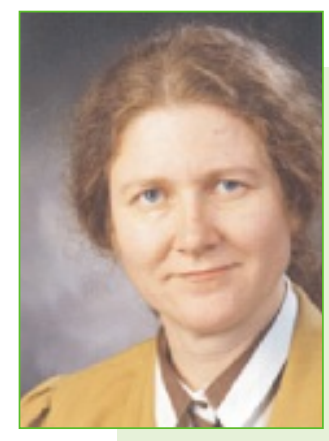

\title{
Strukturwandel in der Medizin
}

Der Wandel in der Medizin vollzieht sich gegenwärtig in hohem Tempo. Während die Akutmedizin aufgrund technischer Fortschritte und auch wegen der Kostenentwicklung ständig im Fokus des allgemeinen Interesses steht, gilt das für andere Felder der Medizin eher nicht. Deshalb sollte man diese aber nicht aus dem Auge verlieren.

So hat sich z.B. in der Rehabilitationsmedizin in den letzten Jahren vieles verändert. Die intensive wissenschaftliche Forschung hat ganz wesentlich zu einer erheblichen Effizienzsteigerung geführt. Von der Akutmedizin unterscheidet sich die Rehabilitationsmedizin jedoch in vielerlei Hinsicht. Ihr Ziel ist die nachhaltige Wiederherstellung von normalen Körperfunktionen und -strukturen und die Überwindung der krankheitsbedingten Beeinträchtigungen bei Aktivitäten und Teilhabe am täglichen Leben. Durch eine erfolgreiche Rehabilitationsmaßnahme sind eine Dosisverminderung oder das Aboder Umsetzen von Dauermedikamenten und -therapien möglich. Hierbei sollte auch an Phytotherapeutika gedacht werden, die bei vielen Indikationen als Ersatz für dann zu stark wirkende oder nebenwirkungsträchtige Medikamente dienen können.

Gegenwärtig gibt es in Deutschland ca. 1270 Vorsorge- oder Rehabilitationseinrichtungen mit insgesamt 175000 Betten mit den Hauptfachrichtungen Innere Medizin (26,2\%), Orthopädie (26,8\%) und Neurologie/Psychiatrie (17,2\%). Die Anzahl der Patienten im Rehabilitationsverfahren betrug 1,82 Mio. im Jahr 2007, in den nächsten Jahren wird sie auf über 2 Mio. ansteigen, da von den Kostenträgern wegen der demografischen Entwicklung vermehrt der Grundsatz "Rehabilitation vor Pflege» umgesetzt werden wird. Rehabilitationsbedürftig sind vor allem ältere Menschen, bei denen oft eine Multimorbidität besteht. Da dementsprechend viele Medikamente eingenommen werden müssen, besteht bei vielen dieser Patienten die Bereitschaft, Phytotherapie anstelle von chemisch definierten Medikamenten einzusetzen, wenn sich dadurch z.B. Nebenwirkungen reduzieren lassen. Bei meinen Konsilen in Rehabilitationskliniken habe ich dies vielfach erfahren.

Bisher war allerdings die dafür erforderliche ärztliche Kompetenz in den Rehabilitationseinrichtungen eher sporadisch vorhanden. Dies dürfte sich in $\mathrm{Zu}$ kunft jedoch deutlich ändern. So sieht die Musterweiterbildungsordnung der Bundesärztekammer von 2008 für das Gebiet Physikalische und Rehabilitative Medizin vor, dass die sekundäre Prävention und die interdisziplinäre Diagnostik, Behandlung und Rehabilitation von körperlichen Beeinträchtigungen, Struktur- und Funktionsstörungen mit konservativen, physikalischen, manuellen und naturheilkundlichen Therapiemaßnahmen sowie die Verfahren der rehabilitativen Intervention erlernt werden.

Aus den Vorgaben der Bundesärztekammer für den Erwerb der Zusatzbezeichnung "Naturheilverfahren» geht hervor, was unter den naturheilkundlichen Therapiemaßnahmen zu verstehen ist. Hier wird neben vielen anderen Verfahren auch die Phytotherapie genannt.

Nach dem Abschluss der Weiterbildung, die vor allem in den stationären Einrichtungen absolviert wird, können die Ärzte für Physikalische und Rehabilitative Medizin in Rehabilitationskliniken, ambulanten Rehabilitationseinrichtungen, Akutkrankenhäusern mit Abteilungen für Frührehabilitation oder für Physikalische Medizin und Rehabilitation, Vertragsarztpraxen, Einrichtungen der Behindertenhilfe und des öffentlichen Gesundheitswesens sowie Einrichtungen der Forschung und Lehre arbeiten.

Im ambulanten Bereich übernehmen sie großenteils das bisherige Tätigkeitsfeld des vertragsärztlich tätigen Orthopäden, sie arbeiten zudem in der Prävention von Erkrankungen durch Aufklärung und durch Anleitung zu gesundheitsförderndem Verhalten, geben Anleitung zu Eigenübungsprogrammen, beraten zu Gesundheitssport und Ernährung und verordnen bei Indikation Funktionstraining. Darüber hinaus koordinieren sie die Zusammenarbeit mit den anderen Gesundheitsberufen wie Krankengymnasten, Ergotherapeuten, Logopäden, Sanitätstechnikern sowie sonstigen sozialen Diensten und Einrichtungen. Arztpraxen für Physikalische und Rehabilitative Medizin können von Kassenpatienten direkt ohne Überweisung aufgesucht werden, für stationäre Abteilungen wird eine Einweisung benötigt.

Wir sollten diese günstige Entwicklung im Gesundheitswesen, die zu einer noch besseren Versorgung gerade der alternden Bevölkerung bei akzeptablen Kosten führen sollte, in jeder Weise unterstützen.

Karin Kraft 